\title{
Human Papillomavirus Infections in Women With and Without Squamous Cell Abnormalities of the Uterine Cervix
}

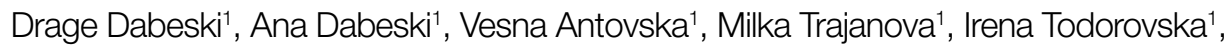 Aneta Sima}

\begin{abstract}
Background: Human papillomavirus infections are one of the most common sexually transmitted infections with viral aetiology. The aim of the study was to confirm the existence of an association between human papillomavirus infection and squamous cell abnormalities of the uterine cervix.

Methods: Cohort study, conducted in the period from January 2017 to June 2018 of 768 sexually active women, age groups of 20 to 59 years, divided into two groups: examined and control, who came to their annual gynaecological exam at University Clinic for Gynaecology and Obstetrics in Skopje. In all patients was done human papillomavirus-deoxyribonucleic testing. Human papillomavirus detection and typing was done using a polymerase chain reaction and reverse hybridisation.

Results: Data analysis showed an association between human papillomavirus infection and squamous cell abnormalities of the uterine cervix $(\mathrm{p}=0.00001)$. Human papillomavirus infection was detected in $22.91 \%$ of all patients, in $75.00 \%$ of patients with abnormal cervical cytology and in $12.50 \%$ of patients with normal cervical cytology. A single human papillomavirus infection was detected in $13.67 \%$ of all patients (in 59.66\% of human papillomavirus positive patients). Mixed human papillomavirus infection was detected in $9.24 \%$ of all patients (in $40.34 \%$ of human papillomavirus positive patients). Human papillomavirus type 16 was the most common genotype with 40.91\%.

Conclusion: This study confirmed that there is an association between human papillomavirus infection and squamous cell abnormalities of the uterine cervix and the young population under the age of 30 years is the most affected.
\end{abstract}

Key words: human papillomavirus, squamous cell abnormalities, uterine cervix.
(1) Department of Gynaecology, University Clinic for Gynaecology and Obstetrics, Skopje, the Republic of North Macedonia.

\section{Correspondence:}

DRAGE DABESK

E: drdabeski@yahoo.com

M: +38970577566

\section{ARTICLE INFO}

Received: 16 April 2019

Revision received: 28 May 2019 Accepted: 1 June 2019

\section{INTRODUCTION}

Human papillomavirus (HPV) infections are one of the most common sexually transmitted infections with viral aetiology. ${ }^{1}$ Epidemiological studies show that $50 \%$ of women are infected with HPV during the first two years of the onset of sex life, about $80 \%$ of sexually active women by the age of 50 will receive genital HPV infection and about $20 \%$ of women are infected with one or more HPV genotypes. ${ }^{2}$ The prevalence of genotype varies depending on the geographical regions. In Europe and North America, HPV-16 is still the most common high-risk genotype. ${ }^{3}$ Young population of women, from 18 to 25, has the highest rate of HPV infection. After the 25th year of life, the incidence of HPV infection is reduced to reach its second highest level after 45 years of life. ${ }^{4}$ The most common risk factor for squamous cell abnormalities of the uterine cervix is infection with HPV, especially with highrisk HPV genotypes. Only persistent, high-risk HPV infection represent a major risk factor for squamous cell abnormalities of the uterine cer-

Copyright $\odot 2019$ Debeski et al. This is an open access article distributed under the Creative Commons Attribution License (CC BY), which permits unrestricted use, distribution, and reproduction in any medium, provided the original work is properly cited. This article should be cited as follows: Dabeski D, Dabeski A, Antovska V, Trajanova M, Todorovska I, Sima A. Human papillomavirus infections in women with and without squamous cell abnormalities of the uterine cervix. Scr Med 2019;50(2):69-76. 
vix. ${ }^{5}$ Deoxyribonucleic acid (DNA) from HPV has been found in $99.7 \%$ of cases of cervical carcinoma. ${ }^{6}$ Detection of HPV can be done using two methods, the first one is direct hybridisation or in situ hybridisation, and the other one is amplification or polymerase chain reaction (PCR). ${ }^{7}$

The aims of the study were: to confirm the existence of an association between HPV-DNA infection and squamous cell abnormalities of the uterine cervix; to detect and typing HPV genotypes, which are the most common causes of squamous cell abnormalities of the uterine cervix; to determine the prevalence of HPV-DNA infection and to determine the most affected age group.

\section{METHODS}

Design of the study: The study is a cohort study.

Material: The material consists of 768 patients aged 20 to 59, divided into two groups: examined and control.

The examined group included 128 sexually active women with an abnormal cervical cytological finding, i.e. a finding of a Papanicolaou (PAP) test that showed a squamous cell abnormalities of the uterine cervix. The control group included 640 sexually active women with a normal cervical cytology finding, i.e. a PAP test.

Exclusion criteria: The study did not include: pregnant women, women with previous surgery of the uterine cervix (cervical vaporisation, carbon dioxide laser vaporization and total abdominal hysterectomy) and also previous abnormal cytological and histopathological findings of the uterine cervix.

Period of realisation: The study was conducted in the period from January 2017 to June 2018.

Location of the study: The study was conducted at the University Clinic for Gynaecology and Obstetrics in Skopje, the Republic of North Macedonia.

Methods of examination: In all women we have done the human papillomavirus-deoxyribonucleic acid (HPV-DNA) testing.

Cytological analysis: All samples for cytological analysis were taken using the Thin Prep PAP test and were analysed in the cytological laboratory at the University Clinic for Gynaecology and Obstetrics in Skopje by a cytopathologist. Cytological results were classified according to the revised Bethesda classification ${ }^{8}{ }^{\prime} 9$ such as: atypical squamous cells of undetermined significance (ASC-US); atypical squamous cells cannot e clude a high-grade squamous intraepithelial lesion (ASC-H); low-grade squamous intraepithelial lesion, LSIL (cervical intraepithelial neoplasia grade 1, CIN 1); high-grade squamous intraepithelial lesion, HSIL (cervical intraepithelial neoplasia grade $2, \mathrm{CIN} 2$, cervical intraepithelial neoplasia grade $3, \mathrm{CIN} 3$, carcinoma in situ, CIS) and invasive squamous cell carcinoma (ISCC).

HPV-DNA testing: Samples from the cervical biopsy for HPV-DNA testing were taken and analysed at the University Clinic for Gynaecology and Obstetrics in Skopje at the Laboratory for HPV testing. HPV detection and typing were used to test the use of multiple polymerase chain reaction (Multiplex PCR) and reverse hybridisation. The results of the HPV DNA test were analysed and demonstrated based on the finding of the presence or absence of DNA from HPV and the specified genotype. ${ }^{10}$ First step in HPV testing was the isolation of DNA from the collected cells from the cervical biopsies. For isolation of DNA series of three paraffin cuts were prepared. Cuts were incubated in $1 \mathrm{ml}$ of xylene, 5 minutes at $55^{\circ} \mathrm{C}$, and centrifuged at 10 ooo $\mathrm{G}$ for five minutes at room temperature. The same procedure was repeated two more times. After careful removal of the remains of xylene, the samples were briefly incubated twice in $1 \mathrm{ml}$ of $100 \%$ ethanol, and centrifuged for 5 minutes at room temperature. After removal of ethanol, short drying followed in air and incubation overnight in buffer with freshly added proteinase $\mathrm{K}$ at $55^{\circ} \mathrm{C}$. The second step was the detection of DNA in HPV by using polymerase chain reaction (PCR). To verify the quality and integrity of the isolated DNA, actually of a present inhibitor, for each sample a reaction of multiplication of the specific primers for beta globin PCO4 and GH2O was first made. Three pairs of primers were used, common to a 
larger number of HPV types: degenerate primers Myo9/My11 and CPI/CPII G and Gp5+/6+. The samples were carried through all reactions with primers specific to high-risk and low-risk HPV genotypes. The third step was genotyping by using reverse hybridisation. It is a method that is based on the hybridisation of specific DNA probes that are immobilised on nitrocellulose or nylon tapes. It is a set of primers (SPF 10) with aim-propagation of the L1 gene on the viral DNA. The product of amplification with SPF promers is the size of $65 \mathrm{bp}$, and allows detection of 25 new genotypes. Denatured biotinylated PCR products are hybridized with specific oligonucleotide probes that are immobilised as parallel lines on membrane strips. After hybridisation and washing with streptavidin, alkaline phosphatase is added, which binds to the biotinylated hybrids formed previously. Incubation with BCIP (5-bromo-4-chloro-3-indolyl-phosphate)/NBT (nitro blue tetrazolium) chromogen

\section{RESULTS}

The distribution of patients by age groups is shown in Table 1. According to the t-test, the percentage difference between the average ages between the two groups was statistically non-significant with p>0.05 (Table 1).

HPV-DNA infection was detected in $22.91 \%$ of all patients, in $75.00 \%$ of patients with abnormal cervical cytology and in $12.50 \%$ of patients with normal cervical cytology. There was a statistically significant relationship between the modalities of HPV-DNA positive and HPV-DNA negative between the examined and the control group (chi-square test $=67.1329, \mathrm{p}<0.00001$, $\mathrm{p}<0.05)$. Data analysis showed: an association gives purple precipitate and the results are interpreted visually.

Statistical analysis: Data were analysed by a specific software for data-bases (Excel). Statistical analysis of the established statistical series was made with the statistical program Statistical Package for Social Sciences (SPSS), version 23.0. The structure of statistical series with attribute sings was analysed with determining the proportions and rates. The structure of numerical signs was analysed by determining the measures of central tendency (arithmetical mean) and measures of dispersion (standard deviation). Analysis of the relationship (the existence of association) between two sets of attribute variables was performed using the Chi-square test and Fisher exact test. Testing the differences between the comparing groups (their distributions, arithmetic environments and proportions) was done using Student's t-test and Chi-square test. Statistical significance was defined as a p value $<0.05$.

between HPV-DNA infection and squamous cell abnormalities of the uterine cervix (Chi-square test $=235.8722, \mathrm{p}=0.00001, \mathrm{p}<0.05)$; an association between the oncogenic potential of the virus and squamous cell abnormalities of the uterine cervix (Fisher exact test $=0.0027, \mathrm{p}<0.05$ ); an increase in the presence of HPV-DNA infection in parallel with an increase in the cytological grade of cervical lesion (Chi-square test $=10.7682$, $\mathrm{p}=0.029296, \mathrm{p}<0.05$ ), and an association between the oncogenic potential of the virus and cytological grade of cervical lesion (Chi-square test $=16.6967, \mathrm{p}=0.000044, \mathrm{p}<0.05$ ) (Table 2).

The incidence of HPV-DNA infection was:

Table 1: Distribution of patients by age groups

\begin{tabular}{|c|c|c|}
\hline Age group & Normal Cytology $(n=640) n(\%)$ & Abnormal cytology $(n=128) n(\%)$ \\
\hline $20-29$ & $130(20.31)$ & $28(21.87)$ \\
\hline $30-39$ & $220(34.38)$ & $38(29.69)$ \\
\hline $40-49$ & $180(28.12)$ & $30(23.44)$ \\
\hline $50-59$ & $110(17.19)$ & $32(25.00)$ \\
\hline Average age & 39.34 & 40.50 \\
\hline Standard deviation & 9.70 & 10.85 \\
\hline \multicolumn{3}{|c|}{ Student's t-test: $p<0.05(p=0.4722, t=0.7204,95 \% C l:-2.01-4.32)$} \\
\hline
\end{tabular}

$\mathrm{n}=$ number of patients 
40.51\% in patients aged 20-29 years; $21.32 \%$ of $30-39$ years; $15.24 \%$ of $40-49$ years; $17.61 \%$ in patients aged 50-59 years. According to the $\mathrm{t}$-test, the percentage difference between incidences of viral infection between the examined and the control group was statistically significant for $\mathrm{p}<0.05(\mathrm{p}=0.0002, \mathrm{t}=8.0032,95 \% \mathrm{CI}$ : 43.9084-82.5816) (Table 3).

A single HPV-DNA infection was detected in $13.67 \%$ of all patients (in $59.66 \%$ of HPV-DNA positive patients). The most common was single HPV-DNA infection with high-risk HPV: 57.39\%. Mixed HPV-DNA infection was detected in $9.24 \%$ of all patients (in $40.34 \%$ of HPV-DNA positive patients). The most common co-infection was high risk - high-risk HPV: $18.18 \%$. According to the t-test, the percentage difference between the modalities of single and mixed HPV-DNA infection between the examined and the control group was statistically non-significant for $\mathrm{p}>0.05(\mathrm{p}=0.7821, \mathrm{t}=0.3157,95 \% \mathrm{CI}$ : $-88.7874-102.8474)$ (Table 4).

Single HPV infection was most frequent in patients over 40 years of age (73.68\%), while mixed HPV infection was most frequent in patients under 30 years of age $(53.12 \%)$ (Table 5 ).

HPV-DNA typing identified a total of $20 \mathrm{HPV}-$

Table 2: Distribution of HPV-DNA infection according to cytological diagnosis

\begin{tabular}{|c|c|c|c|c|c|c|c|c|c|c|}
\hline \multirow{5}{*}{$\begin{array}{l}\text { HPV-DNA } \\
\text { testing }\end{array}$} & \multirow{4}{*}{$\begin{array}{l}\text { All } \\
\text { samples } \\
(n=768)\end{array}$} & \multirow{4}{*}{$\begin{array}{l}\text { Normal } \\
\text { cytology } \\
(n=640)\end{array}$} & \multicolumn{8}{|c|}{ CYTOLOGICAL DIAGNOSIS } \\
\hline & & & \multicolumn{8}{|c|}{ Abnormal cytology } \\
\hline & & & \multirow[t]{2}{*}{$\begin{array}{l}\text { ASC-US } \\
(n=13)\end{array}$} & \multirow[t]{2}{*}{$\begin{array}{l}\text { ASC-H } \\
(n=7)\end{array}$} & \multicolumn{2}{|l|}{$\begin{array}{l}\text { LSIL } \\
(n=31)\end{array}$} & \multicolumn{2}{|c|}{$\begin{array}{l}\text { HSIL } \\
(n=56)\end{array}$} & \multirow[t]{2}{*}{$\begin{array}{l}\text { ISCC } \\
(n=21)\end{array}$} & \multirow[t]{2}{*}{$\begin{array}{l}\text { TOTAL } \\
(n=128)\end{array}$} \\
\hline & & & & & $\begin{array}{l}\mathrm{CIN} 1 \\
(\mathrm{n}=31)\end{array}$ & $\begin{array}{l}\text { CIN 2 } \\
(n=20)\end{array}$ & $\begin{array}{l}\text { CIN 3 } \\
(n=21)\end{array}$ & $\begin{array}{l}\text { In situ SCC } \\
(n=15)\end{array}$ & & \\
\hline & n (\%) & $n(\%)$ & $n(\%)$ & $n(\%)$ & $n(\%)$ & $\mathrm{n}(\%)$ & n (\%) & n (\%) & n (\%) & n (\%) \\
\hline $\begin{array}{l}\text { HPV-DNA } \\
\text { negative }\end{array}$ & $592(77.09)$ & $560(87.50)$ & $7(53.85)$ & $3(42.86)$ & $9(29.03)$ & $4(20.00)$ & $4(19.05)$ & $3(20.00)$ & $2(9.52)$ & $32(25.00)$ \\
\hline $\begin{array}{l}\text { HPV-DNA } \\
\text { positive }\end{array}$ & $176(22.91)$ & $80(12.50)$ & $6(46.15)$ & $4(57.14)$ & $22(70.97)$ & $16(80.00)$ & $17(80.95)$ & $12(80.00)$ & $19(90.48)$ & $96(75.00)$ \\
\hline $\begin{array}{l}\text { High-risk } \\
\text { positive }\end{array}$ & $133(75.57)$ & $60(9.38)$ & $0(0)$ & $3(42.86)$ & 12(38.71) & $11(55.00)$ & 17(80.95) & $12(80.00)$ & 18 (85.71) & 73(57.03) \\
\hline $\begin{array}{l}\text { Low-risk } \\
\text { positive }\end{array}$ & 11 (8.59) & $0(0)$ & 4 (30.77) & 1 (14.28) & $4(12.90)$ & $2(10.00)$ & $0(0)$ & $0(0)$ & $0(0)$ & 11 (8.59) \\
\hline $\begin{array}{l}\text { High and low } \\
\text {-risk positive }\end{array}$ & 72 (40.91) & $60(9.38)$ & $2(23.08)$ & $0(0)$ & $6(19.35)$ & $3(15.00)$ & $0(0)$ & $0(0)$ & $1(4.76)$ & $12(9.37)$ \\
\hline
\end{tabular}

Table 3: Distribution of HPV-DNA infection by age group in 768 patients

\begin{tabular}{|c|c|c|c|c|c|c|c|}
\hline \multirow{3}{*}{$\begin{array}{l}\text { Age } \\
\text { group }\end{array}$} & \multicolumn{3}{|c|}{ NORMAL CYTOLOGY } & \multicolumn{3}{|c|}{ ABNORMAL CYTOLOGY } & \multirow{3}{*}{$\begin{array}{l}\text { Total } \\
\text { HPV-DNA } \\
\text { positive } \\
\text { by age } \\
\text { group } \\
\text { n (\%) }\end{array}$} \\
\hline & $\begin{array}{l}\text { HPV-DNA } \\
\text { positive } \\
(n=80)\end{array}$ & $\begin{array}{l}\text { HPV-DNA } \\
\text { negative } \\
(n=560)\end{array}$ & $\begin{array}{l}\text { TOTAL } \\
(n=640)\end{array}$ & $\begin{array}{l}\text { HPV-DNA } \\
\text { positive } \\
(n=96)\end{array}$ & $\begin{array}{l}\text { HPV-DNA } \\
\text { negative } \\
(n=32)\end{array}$ & $\begin{array}{l}\text { TOTAL } \\
(n=128)\end{array}$ & \\
\hline & n (\%) & n (\%) & n (\%) & n (\%) & n (\%) & n (\%) & \\
\hline $20-29$ & $40(30.77)$ & $90(69.23)$ & $130(20.31)$ & 24 (85.71) & $4(14.29)$ & 28 (21.87) & $64(40.51)$ \\
\hline $30-39$ & $30(13.64)$ & $190(86.36)$ & $220(34.37)$ & $25(65.79)$ & $13(34.21)$ & $38(29.69)$ & 55 (21.32) \\
\hline $40-49$ & $10(5.56)$ & 170 (94.44) & $180(28.13)$ & $22(73.33)$ & $8(26.67)$ & 30 (23.44) & $32(15.24)$ \\
\hline $50-59$ & $0(0)$ & $110(100)$ & $110(17.19)$ & $25(78.12)$ & $7(21.88)$ & $32(25.00)$ & $25(17.61)$ \\
\hline
\end{tabular}

$\mathrm{n}=$ number of patients; HPV-DNA: human papillomavirus deoxyribonucleic acid. 
DNA genotypes, of which 15 were high-risk (HPV DNA-16, -18, -31, -33, -35, -39, -45, -52, $-53,-56,-58-59,-66,-68$, and -73$)$ and 5 lowrisk $(-6,-11,-40,-42$ and -61$)$. The prevalence of $20 \mathrm{HPV}-\mathrm{DNA}$ genotypes in single and mixed HPV-DNA infections according to cytological diagnosis is shown in Table 6. Among high-risk HPV-DNA genotypes, HPV-16 was the most common (40.91\%). Among the low-risk HPVDNA genotypes, the most common was HPV-6 (7.96\%), HPV-16 was most common in patients with high-grade squamous intraepithelial lesion and invasive squamous cell carcinoma, while HPV-6 in patients with low-grade squamous intraepithelial lesion (Table 6).

\section{DISCUSSION}

HPV infections are widespread among people, occur with almost every person in both sexes at a certain period of their life, are usually short-lived, asymptomatic, spontaneously disappearing and only a small percentage of them need treatment. ${ }^{11}$ Seventy-five percentage of the sexually active population, in the course of their lives, was in contact with one or more HPV genotypes. ${ }^{12}$ Depending on the geographical affiliation, the study population and the method used, the frequency of HPV genotypes in various cervical lesions varies considerably. In 1996, the World Health Association recognized the importance of HPV for cervical cancer. ${ }^{6}$

Table 4: Distribution of single and mixed HPV-DNA infections in 176 HPV-DNA positive patients

\begin{tabular}{|c|c|c|c|c|c|c|c|c|c|c|}
\hline \multirow{5}{*}{$\begin{array}{l}\text { Type of } \\
\text { HPV-DNA } \\
\text { infection }\end{array}$} & \multirow{5}{*}{$\begin{array}{l}\text { Total } \\
\text { HPV-DNA } \\
\text { positive } \\
\text { (n=176) } \\
\text { n (\%) }\end{array}$} & \multicolumn{9}{|c|}{ CYTOLOGICAL DIAGNOSIS } \\
\hline & & \multirow{3}{*}{$\begin{array}{l}\text { Normal } \\
\text { cytology } \\
(n=80)\end{array}$} & \multicolumn{8}{|c|}{ Abnormal cytology } \\
\hline & & & \multirow{3}{*}{ 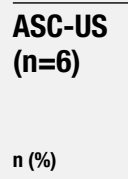 } & \multirow{3}{*}{$\begin{array}{l}\begin{array}{l}\text { ASC-H } \\
(n=4)\end{array} \\
n(\%)\end{array}$} & \multirow{3}{*}{$\begin{array}{l}\text { LSIL }(n=22 \\
\text { CIN } 1 \\
(n=22) \\
n(\%)\end{array}$} & \multirow{3}{*}{$\begin{array}{l}\text { CIN } 2 \\
(n=16) \\
n(\%)\end{array}$} & \multicolumn{2}{|c|}{ HSIL $(n=45)$} & \multirow{3}{*}{$\begin{array}{l}\begin{array}{l}\text { ISCC } \\
(n=19)\end{array} \\
n(\%)\end{array}$} & \multirow{3}{*}{$\begin{array}{l}\text { TOTAL } \\
(\mathrm{n}=96)\end{array}$} \\
\hline & & & & & & & $\begin{array}{l}\text { CIN 3 } \\
(n=17)\end{array}$ & $\begin{array}{l}\text { In situ SCC } \\
(n=12)\end{array}$ & & \\
\hline & & $n(\%)$ & & & & & $n(\%)$ & $n(\%)$ & & \\
\hline Single & $105(59.66)$ & $50(62.50)$ & $1(16.67)$ & $1(25.00)$ & $10(45.45)$ & $8(50.00)$ & $12(70.59)$ & $10(83.33)$ & $13(68.42)$ & $55(57.29)$ \\
\hline Single high-risk & $101(57.39)$ & $50(62.50)$ & $0(0)$ & $0(0)$ & $8(36.36)$ & $8(50.00)$ & $12(70.59)$ & $10(83.33)$ & $13(68.42)$ & $51(53.12)$ \\
\hline Single low-risk & $4(2.27)$ & $0(0)$ & $1(16.67)$ & $1(25.00)$ & $2(9.09)$ & $0(0)$ & $0(0)$ & $0(0)$ & $0(0)$ & $4(4,17)$ \\
\hline Mixed & $71(40.34)$ & $30(37.50)$ & $5(83.33)$ & $3(75.00)$ & $12(54.55)$ & $8(50.00)$ & $5(29.41)$ & $2(16.67)$ & $6(31.58)$ & $41(42.71)$ \\
\hline $\begin{array}{l}\text { Mixed high-risk- } \\
\text { high risk }\end{array}$ & $32(18.18)$ & $10(12.50)$ & 1 (16.67) & $1(25.00)$ & $3(13.64)$ & $6(37.50)$ & $4(23.53)$ & $2(16.67)$ & $5(26.32)$ & 22 (22.92) \\
\hline $\begin{array}{l}\text { Mixed high-risk- } \\
\text { low risk }\end{array}$ & 31 (17.61) & $20(25.00)$ & $3(50.00)$ & $1(25.00)$ & $3(13.64)$ & $2(12.50)$ & $1(5.88)$ & $0(0)$ & $1(5.26)$ & $11(11.46)$ \\
\hline $\begin{array}{l}\text { Mixed low-risk- } \\
\text { low risk }\end{array}$ & $8(4.55)$ & $0(0)$ & 1 (16.67) & $1(25.00)$ & $6(27.27)$ & $0(0)$ & $0(0)$ & $0(0)$ & $0(0)$ & $8(8.33)$ \\
\hline
\end{tabular}

$\mathrm{n}=$ number of patients; ASC-US, atypical squamous cells of undetermined significance; ASC-H: atypical squamous cells-cannot exclude high-grade squamous intraepithelial lesion; LSIL: low-grade squamous intraepithelial lesion; HSIL: high-grade squamous intraepithelial lesion; ISCC: invasive squamous cell carcinoma; CIN: cervical intraepithelial neoplasia; HPV-DNA: human papillomavirus deoxyribonucleic acid.

Table 5: Distribution of single and mixed HPV-DNA infections by age group in 176 HPV-DNA positive patients

\begin{tabular}{|c|c|c|c|c|c|c|c|}
\hline \multirow{3}{*}{$\begin{array}{l}\text { Age } \\
\text { group }\end{array}$} & \multicolumn{3}{|c|}{ NORMAL CYTOLOGY } & \multicolumn{3}{|c|}{ ABNORMAL CYTOLOGY } & \multirow{2}{*}{$\begin{array}{l}\text { All } \\
\text { HPV-DNA } \\
\text { positive } \\
(n=176)\end{array}$} \\
\hline & $\begin{array}{l}\text { Single } \\
\text { HPV-DNA } \\
\text { infection } \\
(n=50)\end{array}$ & $\begin{array}{l}\text { Mixed } \\
\text { HPV-DNA } \\
\text { infection } \\
(n=30)\end{array}$ & $\begin{array}{l}\text { TOTAL } \\
(n=80)\end{array}$ & $\begin{array}{l}\text { Single } \\
\text { HPV-DNA } \\
\text { infection } \\
(n=55)\end{array}$ & $\begin{array}{l}\text { Mixed } \\
\text { HPV-DNA } \\
\text { infection } \\
(\mathrm{n}=41)\end{array}$ & $\begin{array}{l}\text { TOTAL } \\
(n=96)\end{array}$ & \\
\hline & $n(\%)$ & n (\%) & n (\%) & $\mathrm{n}(\%)$ & $\mathrm{n}(\%)$ & n (\%) & n (\%) \\
\hline $20-29$ & $20(50.00)$ & $20(50.00)$ & $40(50.00)$ & $10(41.67)$ & $14(58.33)$ & $24(25.00)$ & $64(36.36)$ \\
\hline $30-39$ & $20(66.67)$ & $10(33.33)$ & $30(37.50)$ & $13(52.00)$ & $12(48.00)$ & $25(26.04)$ & $55(31.25)$ \\
\hline $40-49$ & $10(100)$ & $0(0)$ & $10(12.50)$ & $14(63.64)$ & $8(36.36)$ & $22(22.92)$ & $32(18.18)$ \\
\hline $50-59$ & $0(0)$ & $0(0)$ & $0(0)$ & $18(72.00)$ & $7(28.00)$ & $25(26.04)$ & $25(14.21)$ \\
\hline
\end{tabular}

$\mathrm{n}=$ number of patients; HPV-DNA: human papillomavirus deoxyribonucleic acid. 
Table 6: The prevalence of HPV-DNA genotypes according to cytological diagnosis

\begin{tabular}{|c|c|c|c|c|c|c|c|c|c|c|c|}
\hline \multirow{5}{*}{ 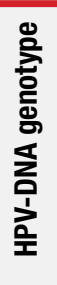 } & \multirow{5}{*}{ 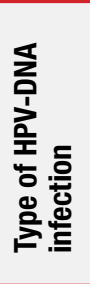 } & \multirow{5}{*}{$\begin{array}{l}\begin{array}{l}\text { All } \\
\text { HPV-DNA } \\
\text { positive } \\
(n=176)\end{array} \\
n(\%)\end{array}$} & \multicolumn{9}{|c|}{ CYTOLOGICAL DIAGNOSIS } \\
\hline & & & \multirow{4}{*}{$\begin{array}{l}\begin{array}{l}\text { Normal } \\
\text { cytology } \\
(\mathrm{n}=80)\end{array} \\
\mathrm{n}(\%)\end{array}$} & \multicolumn{8}{|c|}{ Abnormal cytology } \\
\hline & & & & \multirow{3}{*}{$\begin{array}{l}\text { ASC-US } \\
(n=6)\end{array}$} & \multirow{3}{*}{$\begin{array}{l}\begin{array}{l}\text { ASC-H } \\
(n=4)\end{array} \\
n(\%)\end{array}$} & \multirow{3}{*}{$\begin{array}{l}\begin{array}{l}\text { LSIL } \\
(\mathbf{n}=22)\end{array} \\
\text { CIN 1 } \\
(n=22) \\
n(\%)\end{array}$} & \multicolumn{2}{|c|}{ HSIL ( $n=45)$} & & \multirow[t]{2}{*}{$\begin{array}{l}\text { ISCC } \\
(n=19)\end{array}$} & \multirow[t]{3}{*}{$\begin{array}{l}\text { TOTAL } \\
(n=96)\end{array}$} \\
\hline & & & & & & & $\begin{array}{l}\text { CIN 2 } \\
(n=16)\end{array}$ & $\begin{array}{l}\text { CIN 3 } \\
(n=17)\end{array}$ & $\begin{array}{l}\text { In situ SCC } \\
(n=12)\end{array}$ & & \\
\hline & & & & & & & $n(\%)$ & $n(\%)$ & $n(\%)$ & $n(\%)$ & \\
\hline \multirow{2}{*}{16} & Single & $45(25.57)$ & $20(25.00)$ & - & - & $1(4.54)$ & $4(25.00)$ & $9(52.94)$ & $5(41.67)$ & $6(31.58)$ & $25(26.04)$ \\
\hline & Mixed & $27(15.34)$ & $10(12.50)$ & $1(16.67)$ & $2(50.00)$ & $1(4.54)$ & $3(18.75)$ & $3(17.65)$ & $3(25.00)$ & $4(21.05)$ & $17(17.71)$ \\
\hline \multirow{2}{*}{18} & Single & $3(1.70)$ & - & - & - & - & - & - & - & $3(15.79)$ & $3(3.12)$ \\
\hline & Mixed & $17(9.66)$ & $10(12.50)$ & - & - & $1(4.54)$ & $1(6.25)$ & $2(11.76)$ & - & $3(15.79)$ & $7(7.29)$ \\
\hline \multirow[b]{2}{*}{31} & Single & $8(4.55)$ & - & - & - & - & $1(6.25)$ & $3(17.65)$ & $3(25.00)$ & $1(5.26)$ & $8(8.33)$ \\
\hline & Mixed & $7(3.98)$ & - & - & - & 14.54) & $1(6.25)$ & $1(5.88)$ & $2(16.67)$ & $2(10.53)$ & $7(7.29)$ \\
\hline \multirow{2}{*}{33} & Single & $2(1.14)$ & - & - & - & - & - & $1(5.88)$ & $1(8.33)$ & - & $2(2.08)$ \\
\hline & Mixed & $5(2.84)$ & - & $1(16.67)$ & - & - & $2(12.50)$ & - & - & $2(10.53)$ & $5(5.21)$ \\
\hline \multirow[b]{2}{*}{35} & Single & $1(0.57)$ & - & - & $1(25.00)$ & - & - & - & - & - & $1(1.04)$ \\
\hline & Mixed & $14(7.95)$ & $10(12.50)$ & - & $1(25.00)$ & $1(4.54)$ & $1(6.25)$ & $1(5.88)$ & - & - & $4(4.17)$ \\
\hline \multirow{2}{*}{39} & Single & $10(5.68)$ & $10(12.50)$ & - & - & - & - & - & - & - & - \\
\hline & Mixed & $1(0.56)$ & - & - & - & $1(4.54)$ & - & - & - & - & $1(1.04)$ \\
\hline \multirow{2}{*}{45} & Single & $7(3.98)$ & - & - & - & - & $1(6.25)$ & - & $2(16.67)$ & $4(21.05)$ & $7(7.29)$ \\
\hline & Mixed & $2(1.14)$ & - & - & - & - & - & - & - & $2(10.53)$ & $2(2.08)$ \\
\hline \multirow{2}{*}{52} & Single & $1(0.57)$ & - & $1(16.67)$ & - & - & - & - & - & - & $1(1.04)$ \\
\hline & Mixed & $14(7.95)$ & $10(12.50)$ & - & - & $1(4.54)$ & $1(6.25)$ & $2(11.76)$ & - & - & $4(4.17)$ \\
\hline \multirow[b]{2}{*}{53} & Single & - & - & - & - & - & - & - & - & - & - \\
\hline & Mixed & $1(0.57)$ & - & $1(16.67)$ & - & - & - & - & - & - & $1(1.04)$ \\
\hline & Single & $2(1.14)$ & - & $1(16.67)$ & - & $1(4.54)$ & - & - & - & - & $2(2.08)$ \\
\hline 56 & Mixed & $3(1.70)$ & - & 1 (16.67) & - & $1(4.54)$ & - & $1(5.88)$ & - & - & $3(3.12)$ \\
\hline & Single & $20(11.36)$ & $20(25.00)$ & - & - & - & - & - & - & - & - \\
\hline 58 & Mixed & $1(0.57)$ & - & - & - & - & $1(6.25)$ & - & - & - & $1(1.04)$ \\
\hline & Single & - & - & - & - & - & - & - & - & - & - \\
\hline 59 & Mixed & $2(1.14)$ & - & - & - & $2(9.09)$ & - & - & - & - & $2(2.08)$ \\
\hline & Single & $2(1.14)$ & - & - & - & $2(9.09)$ & - & - & - & - & $2(2.08)$ \\
\hline 66 & Mixed & $1(0.57)$ & - & - & - & $1(4.54)$ & - & - & - & - & $1(1.04)$ \\
\hline & Single & - & - & - & - & - & - & - & - & - & - \\
\hline 68 & Mixed & $13(7.39)$ & $10(12.50)$ & - & - & $1(4.54)$ & $1(6.25)$ & $1(5.88)$ & - & - & $3(3.12)$ \\
\hline & Single & - & - & - & - & - & - & - & - & - & - \\
\hline 73 & Mixed & $2(1.14)$ & - & - & - & $1(4.54)$ & - & - & $1(1.88)$ & - & $2(2.08)$ \\
\hline & Single & $1(0.57)$ & - & $1(16.67)$ & - & - & - & - & - & - & $1(1.04)$ \\
\hline 6 & Mixed & $13(7.39)$ & $1(1.25)$ & 4 (66.67) & $3(75.00)$ & 4 (18.18) & - & $1(5.88)$ & - & - & 13 (13.54) \\
\hline & Single & $1(0.57)$ & - & - & - & $1(4.54)$ & - & - & - & - & $1(1.04)$ \\
\hline 11 & Mixed & $8(4.54)$ & - & - & - & $8(36.36)$ & - & - & - & - & $8(8.33)$ \\
\hline & Single & - & - & - & - & - & - & - & - & - & - \\
\hline 40 & Mixed & $2(1.14)$ & - & - & - & $2(9.09)$ & - & - & - & - & $2(2,08)$ \\
\hline & Single & $2(1.14)$ & - & 1 (16.67) & - & $1(4.54)$ & - & - & - & - & $2(2.08)$ \\
\hline 42 & Mixed & $2(1.14)$ & $1(1.25)$ & - & - & - & - & - & - & $1(5.26)$ & $1(4.76)$ \\
\hline & Single & - & - & - & - & - & - & - & - & - & \\
\hline 61 & Mixed & $4(2.27)$ & - & 2 (33.33) & $1(25.00)$ & - & - & $1(5.88)$ & - & - & $4(4.17)$ \\
\hline
\end{tabular}

$\mathrm{n}=$ number of patients; ASC-US: atypical squamous cells of undetermined significance; ASC-H: atypical squamous cells-cannot exclude high-grade squamous intraepithelial lesion; LSIL: low-grade squamous intraepithelial lesion; HSIL: high-grade squamous intraepithelial lesion; ISCC: invasive squamous cell carcinoma; CIN: cervical intraepithelial neoplasia; HPV-DNA: human papillomavirus deoxyribonucleic acid.

In this study, HPV-DNA infection was detected in $75.00 \%$ of patients in the investigated group and in $12.50 \%$ of patients in the control group. This relatively high percentage of HPVDNA infection in patients with squamous cell abnormalities of the uterine cervix corresponds with some previously published studies; in the study of Mazarico et al. from 2012, HPV-DNA infection was detected in $73.20 \%$ of women with squamous cell abnormalities of the uterine cervix, ${ }^{13}$ while in the study of Pista et al. from
2013, HPV-DNA infection was detected in $77.4 \%$ of study women. ${ }^{14}$ The detected prevalence of $12.50 \%$ of HPV-DNA infection of the uterine cervix in patients with a normal cytological finding in our study is in accordance with the estimated global prevalence of $10-12 \%$, with variations of $1-26 \%$ depending on the geographical affiliation and age. ${ }^{15}$ The British meta-analysis of Anderson et al. since 2013, women with normal cervical cytological findings have shown a prevalence of HPV-DNA infection of $12.00 \% .{ }^{16}$ An estimat- 
ed prevalence of $\mathbf{1 1 . 2} \%$ in women with normal cervical cytology is shown in the Chinese study of Bao et al. from 2008, worked on 2902 women. ${ }^{17}$ An identical prevalence (11.1\%) is shown in the Chilean study of Balanda et al. from 2014$2015 .^{18}$

In this study, a significant association was found between the presence of HPV-DNA infection and the incidence of squamous cell abnormalities of the uterine cervix $(p=0.00001)$. The high percentage of high-risk HPV-DNA genotypes in severe dysplasia (80.95\%), and in situ squamous cell carcinoma $(80.00 \%)$ and invasive squamous cell carcinoma (90.48\%), once again confirms the strong association between the oncogene potential of the virus and the development of squamous intraepithelial lesions and squamous invasive carcinoma of the cervix $(\mathrm{p}<0.05)$. The relationship between high-risk and low-risk HPV-genotypes in HPV-DNA positive patients was $75.57 \%: 8.59 \%$. In the Spanish study of Garcia-Garcia from 2010, that ratio was 79.80\%:19.70\%.19

The highest frequency of HPV-DNA infection was found in patients under 30 years of age (40.51\%), while the lowest in patients aged 40 to $49(15.25 \%)$. Our results correspond to the results of some previously published studies: in the Italian study of Agodi et al. from 2009, the highest frequency of HPV-DNA infection was detected in patients under the age of 25 (73.2\%);20 American study of Evans from 2006 showed the highest frequency of HPV-DNA infection in patients under the age of $30(96.00 \% ; 458 / 475) ; 21$ an identical percentage of HPV-DNA infection (96.00\%), also showed the study of Hariri et al. from 2012, worked on 3058 American women aged 18 to 39.22

In this study, mixed HPV-DNA infection was found in $9.24 \%$ of all patients, in $40.34 \%$ of HPV-DNA positive. This percentage of prevalence of mixed HPV-DNA infections corresponds to previously published studies of: Sandri et al. from 2009 (43.00\%), 23 Cuschieri et al. from 2001 (43.30\%), ${ }^{24}$ Vujosevic et al. from $2012(42.00 \%) .25$

In the group of patients under 30 years of age, the highest frequency of mixed HPV infection was detected (53.12\%). These high frequencies in the young population can be explained by their sexual behavior, that is, promiscuity. In a Serbian study by Kovacevic G. et al. from 2016, performed in students from the University of Novi Sad, mixed HPV infection was detected in
49.4\%. ${ }^{26}$ Relatively high rates of mixed HPV infection among the young population were also detected in the Bulgarian studies of Grozdanovin $2014(63.00 \%)^{27}$ and Kovachev in 2013 (53.00\%), 28 as well as in the Romanian study of Moga in 2014 (54.93\%). ${ }^{29}$

HPV-16 was the most common genotype with $40.91 \%$. In the retrospective study of Andonovska in 2014, performed in 7,411 women, the following distribution of the most common ge otypes was detected: HPV-16 (23.39\%), HPV31 (10.68\%), HPV-53 (10.60\%) and HPV-18 (6.19\%). ${ }^{30}$ In the study of Stojanovska et al. (2009), performed in 6,988 patients, the following distribution of the most common genotypes was detected: HPV-16 (32.1\%), HPV-31 (14\%), HPV-53 (12.6\%), HPV-18 9.9\%), HPV-58 (5\%), etc. ${ }^{31}$ At the same time, in the study of Duvlis in 2000, performed in patients from the Republic of North Macedonia, the following distribution of the most common genotypes was detected: HPV-16 (27.5\%), HPV-31 (13.1\%), HPV-66 (10.3\%), HPV-6 (9.4\%), HPV-18 (8.4\%), etc.. ${ }^{32}$ In the four Macedonian studies, the most common HPV genotype was HPV-16, but there are deviations in the distribution of the other most common HPV genotypes. The high percentage of HPV-16 in severe dysplasia (70.59\%), in in situ squamous cell carcinoma (66.67\%) and invasive squamous carcinoma (52.63\%), distinguishes HPV-16 as a high risk genotype with the highest oncogenic potential. It is confirmed by this study that: there is an association between HPV-DNA infection and squamous cell abnormalities of the uterine cervix; the young population under the age of 30 years is the most affected and the HPV-16 is the most common genotype in our environment.

\section{CONCLUSION}

It is confirmed by this study that: there is an association between HPV-DNA infection and squamous cell abnormalities of the uterine cervix; the young population under the age of 30 years is the most affected and the HPV-16 is the most common genotype in our environment.

\section{ACKNOWLEDGEMENTS}

None.

\section{CONFLICT OF INTEREST}

None. 


\section{REFERENCES}

1. Pirrota M, Ung L, Stein A, Mast TC. The psychosocial burden of human papillomavirus related disease and screening interventions. Sex Transm Infect 2009;85:508-13.

2. Pierce CM, Menezes LJ, Paskett ED, Giuliano AR. Prevention of invasive cervical cancer in the United States: past, present and future. Cancer Epidemiol Biomarkers Prev 2012;21(9):1402-8.

3. Cuzick J, Clavel C, Petry K, Meijer CJLM, Hoyer H, Ratnam S, Iftner T. Overview of the European and North American studies on HPV testing in primary cervical cancer screening. Int J Cancer 2006;119(5):1095-101.

4. Lau S, Franco EL. Management of low-grade cervical lesions in young women. Can Med Assoc J 2005;173(7):771-4.

5. Bosch FX, Lorincz A, Munoz N, Meijer CJLM, Shah $\mathrm{KV}$. The causal relation between human papillomavirus and cervical cancer. J Clin Pathol 2002;55(4):24465 .

6. zur Hausen H. Papillomaviruses and cancer: from basic studies to clinical application. Nature Rev Cancer 2002; 2(5):342-50.

7. Brink AATP, Snijders PJF, Meijer CJLM. HPV detection methods. Dis Markers 2007;23(4):273-81.

8. Zerat L. La nouvelle terminologie de Bethesda: quells-changements. Rev Prat Gynecol Obstet 2002;Numero Special: 3-10.

9. Solomon D, Davey D, Kurman R, Moriary A, O'Connor D, Prey M, et al. The 2001 Bethesda System: terminology for reporting results of cervical cytology. JAMA 2002;287(16):2114-9.

10. Franco EL. Primary screening of cervical cancer with human papillomavirus tests. JNCI Monographs 2003;2003(31):89-96.

11. 11. Sweet RL, Gibbs RS. Infectious disease of the female genital tract. Philadelphia, PA, USA: Wolters Kluwer Health, Lippincott Williams \& Wilkins; 2009.

12. Vieira L, Almeida A. The cytology and DNA detection by the PapilloCheck test in the diagnosis of human papillomavirus infection. Eur J Microbiol Immunol 2013;3(1):61-7.

13. Mazarico E, Gonzalez-Bosquet E. Prevalence of infection by different genotypes of human papillomavirus in women with cervical pathology. Gynecol Oncol 2012;125(1):181-5.

14. Pista A, de Oliveira CF, Lopes C, Cunha MJ; CLEOPATRE Portugal Study Group. Human papillomavirus type distribution in cervical intraepithelial neoplasia grade 2/3 and cervical cancer in Portugal: a CLEOPATRE II Study. Int J Gynecol Cancer 2013;23(3):500-6.

15. Bruni L, Diaz M, Castellsague X, Ferrer E, Bosch FX, de Sanjose S. Cervical human papillomavirus prevalence in 5 continents: meta-analysis of 1 million women with normal cytological findings. J Infect Dis 2010;202(12):1789-99.

16. Anderson L, O'Rorke M, Jamison J, Wilson R, Gavin A. Prevalence of human papillomavirus in women attending cervical screening in the UK and Ireland: new data from Northern Ireland and a systematic review and meta-analysis. J Med Virol 2013;85(2):295-308.

17. Bao YP, Li N, Smith JS, Qiao YL. Human papillomavirus type-distribution in the cervix of Chinese women: a meta-analysis. Int J STD AIDS 2008;19(2):106-11.

18. Balanda M, Quiero A, Vergara N, Espinoza G, Martin HS, Rojas G, et al. Prevalence of human papillomavirus infection among women presenting for cervical cancer screening in Chile, 2014-2015. Med Microbiol Immunol 2016;205(6):585-94.
19. Garcia-Garcia JA, Perez-Valles A, Martorell M, Gomez B, Gomez-Cabrero D, Soler F, et al. Distribution of human papillomavirus types in women from Valencia, Spain, with abnormal cytology. Acta Cytol 2010;54(2):159-64.

20. Agodi A, Barchitta M, La Rosa N, Cipresso R, Guarnaccia M, Castiqlione MG, et al. Human papillomavirus infection: low-risk and high-risk genotypes in women in Catania, Sicily. Int $\mathrm{J}$ Gynecol Cancer 2009;19(6):1094-8.

21. Evans MF, Adamson CS, Papillo JL, St John TL, Leiman G, Cooper K. Distribution of human papillomavirus types in ThinPrep Papanicolaou tests classified according to the Bethesda 2001 terminology and correlations with patient age and biopsy outcomes. Cancer 2006;106(5):1054-64.

22. Hariri S, Unger ER, Powell SE, Bauer HM, Bennett NM, Bloch KC, et al; HPV-IMPACT Working Group. Human papillomavirus genotypes in high-grade cervical lesions in the United States. J Infect Dis 2012;206(12):1878-86.

23. Sandri MT, Riggio D, Salvatici M, Passerin R, Zorzino L, Boveri S, et al. Typing of human papillomavirus in women with cervical lesions: prevalence and distribution of different genotypes. J Med Virol 2009;81(2):271-7.

24. Cuschieri KS, Cubie HA, Whitley MW, Seagar AL, Arends MJ, Moore C, et al. Multiple high risk HPV infections are common in cervical neoplasia and young women in a cervical screening population. J Clin Pathol 2004;57(1):68-72.

25. Vujošević D, Vuksanović V, Poljak M, Jokmanović N. Human papillomavirus genotype spectrum in studied group of Montenegrin women. Acta Medica (Hradec Kralove) 2012;55(3):130-2.

26. Kovačević G, Jovanović-Galović A, Petrović V, Vinarž Z, G Marinković G, Brašanac B, et al. Human papillomavirus infection prevalence in female university students in Novi Sad, Serbia. Srp Arh Celok Lek 2016;144(5-6):300-6.

27. Grozdanov P, Zlatkov V, Ganchev G, Karagiosov I, Toncheva D, Galabov AS. HPV prevalence and type distribution in women with normal or abnormal Pap smear in Bulgaria. J Med Virol 2014;88(11):1905-10.

28. 28. Kovachev S, Slavov V, Slavova K. Prevalence of human papillomavirus infection in women in some cities and regions of Bulgaria. J Med Virol 2013;85(9):157784.

29. Moga MA, Irimi, M, Oanta A, Pascu A, Burtea V. Type-specific Prevalence of Human Papillomavirus by Cervical Cytology among Women in Brasov, Romania. Asian Pac J Cancer Prev 2014;15(16):6887-92.

30. Andonovska J. Genotyping distribution and prevalence of the HPV infection in Macedonia. Int J Infect Dis 2014;21(1):398.

31. Stojanovska V, Panov S, Bašeska N, Josifovska S, Dimitrov G, Petanovski Z, et al. [Prevalence and distribution of human papillomavirus infections in clinically suspicious cervical lesions]. Mak Med Pregled 2009;63(1):17-24. Macedonian.

32. Duvlis S. [Genotyping of human papillomavirus (HPV) in female population of Republica of Macedonia]. [master's thesis]. [Skopje (MK)]: Ss. Cyril and Methodius University; 200o. Macedonian. 